\title{
EARLINET SINGLE CALCULUS CHAIN: NEW PRODUCTS OVERVIEW
}

\author{
Giuseppe D'Amico ${ }^{1,{ }^{*}}$, Ina Mattis ${ }^{2}$, Ioannis Binietoglou ${ }^{3,5}$, Holger Baars ${ }^{4}$, Lucia Mona ${ }^{5}$, \\ Francesco Amato ${ }^{1}$, Panos Kokkalis ${ }^{5}$, Alejandro Rodríguez-Gómez ${ }^{6}$, Ourania Soupiona ${ }^{7}$ \\ and Voudouri Kalliopi-Artemis ${ }^{8}$ \\ ${ }^{1}$ Consiglio Nazionale delle Ricerche, Istituto di Metodologie per l'Analisi Ambientale \\ (CNR-IMAA), Potenza Italy, *Email:giuseppe.damico@imaa.cnrit \\ ${ }^{2}$ Deutscher Wetterdienst, Meteorologisches Observatorium Hohenpeißenberg, Germany \\ ${ }^{3}$ National Institute of R\&D for Optoelectronics INOE, Bucharest, Romania \\ ${ }^{4}$ Leibniz Institute for Tropospheric Research (TROPOS), Leipzig, Germany \\ ${ }^{5}$ National Observatory of Athens, Athens, Greece \\ ${ }^{6}$ BarcelonaTech University (UPC), Barcelona, Spain \\ ${ }^{7}$ Laser Remote Sensing Unit, National Technical University of Athens, Physics Dept., Greece \\ ${ }^{8}$ Laboratory of Atmospheric Physics, Aristotle University of Thessaloniki, Greece
}

\begin{abstract}
The Single Calculus Chain (SCC) is an automatic and flexible tool to analyze raw lidar data using EARLINET quality assured retrieval algorithms. It has been already demonstrated the SCC can retrieve reliable aerosol backscatter and extinction coefficient profiles for different lidar systems. In this paper we provide an overview of new SCC products like particle linear depolarization ratio, cloud masking, aerosol layering allowing relevant improvements in the atmospheric aerosol characterization.
\end{abstract}

\section{INTRODUCTION}

The SCC is the standard EARLINET (European Aerosol Research Lidar Network) tool for the analysis of lidar data providing highquality aerosol properties at network level. The SCC has been installed on a centralized server where raw data measured by all EARLINET systems can be submitted and automatically analyzed. Moreover, the SCC is highly configurable and can be easily adapted to analyze data coming from new lidar systems. In particular, using a user-friendly web interface it is possible to change all instrumental and configuration parameters to be used in the analysis. The products of the SCC are all quality certified according to the EARLINET quality assurance program. The SCC can provide different levels of output: pre-processed signals (range-corrected lidar signals corrected for all instrumental effects) and aerosol optical products (aerosol backscatter and extinction coefficient profiles). The SCC comprises two main calculus modules: the ELPP (EARLINET Lidar Pre-Processor) module which accepts as input the raw lidar data and produces the preprocessed signals [1] and the ELDA (EARLINET Lidar Data Analyzer) module which takes as inputs the outputs of the ELPP module and generates optical products like particle extinction and backscatter coefficient [2]. The actions of the two modules are automatically synchronized and coordinated by another module called SCC daemon. All parameters required by the ELPP and ELDA modules are stored in an efficient way in an MySQL database. The SCC has been validated by [2] using synthetic lidar signals used during the EARLINET algorithm inter-comparison exercise and by [3] using real lidar data. Such validations show good SCC performance in analyzing specific measurements of several co-located lidar systems as well as in case of statistical analysis made by using long-term data set.

The development of the SCC is continuing and new important features are going to be included in the framework of the ACTRIS-2 project. In this paper we provide an overview on these new SCC products.

\section{NEW SCC PRODUCTS}

The SCC can deliver quality assured particle extinction and backscatter coefficient profiles. 
It is well known that this set of optical parameters can already provide a quite complete characterization of atmospheric aerosols especially in case of multi-wavelength Raman lidar measurements. Anyway, to further improve the SCC capabilities in characterizing atmospheric particles, the following new products have been included: 1) particle linear depolarization ratio, 2) cloud masking, and 3) aerosol layering. The calculation of particle linear depolarization ratio allows a better differentiation between spherical and not spherical particles making easier to discriminate for example dust or ash from marine or sulfate aerosol types. The cloud masking is another important improvement because the quality of the SCC optical products cannot be assured, if there are signatures of low-level clouds in the raw lidar time series. Without a cloud masking module it is an user responsibility to not submit raw datafile contaminated by low-level clouds and this makes the process of submission to the SCC not fully automatable. Finally, the automatic characterization of the atmospheric aerosol layers in terms of their optical and geometrical properties, makes feasible the provision of the SCC products to external algorithms related for example to micro-physical retrievals or aerosol typing.

\subsection{Particle Linear Depolarization Ratio}

The Volume Linear Depolarization Ratio (VLDR) and Particle Linear Depolarization Ratio (PLDR) are of great importance for the characterization of atmospheric aerosols especially in terms of spherical and not-spherical scatterers. Before the implementation of the calculation of the PLDR and VLDR products in the SCC it is required to define quality assured procedures for the retrieval of these important parameters. Despite the apparent simplicity, there are several instrumental effects that need to be taken into account to deliver reliable PLDR values. Such effects are highly system dependent and require dedicated measurements to fully characterize all the optics of lidar from polar- ization point of view. In what it follows, we briefly summarize the basic aspects regarding the implementation of the PLDR and VLDR in the SCC. The full theoretical background behind what has been implemented is described in details by [4]. The first critical point is the calibration of the polarization sensitive channels involved in the calculation of the VLDR (and consequently of the PLDR). As, in general, the total efficiency of the polarization channels perpendicular and parallel to the direction of the polarization of the emitted light (supposed to be linear polarized) are different, accurate values of the gain ratio $\eta^{*}$ of these two channels are required. The calibration procedures implemented in the SCC are based on +45 or $\Delta 90$ calibration methods [4] and are made by submitting to the SCC a raw calibration dataset. Calibration based on Rayleigh method is not implemented because it is considered not enough accurate (small strongly depolarizing aerosol layer within the assumed aerosol-free atmospheric region could lead to major deviations from the assumed molecular depolarization value). Once the value of $\eta^{*}$ is determined it is possible to calculate the "apparent" VLDR:

$$
\delta^{*}=\frac{K}{\eta^{*}} \frac{I_{R}}{I_{T}}
$$

where the factor $K$ is a calibration correction factor defined in [4] and $I_{R}$ and $I_{T}$ are the intensity or the radiation reflected and transmitted by the polarization sub-system. For ideal systems for which the cross polarized channel is reflected and the parallel one is transmitted, $\delta^{*}$ is equal to the VLDR $\delta$. For real systems the relationship between $\delta^{*}$ and $\delta$ is more complex as it needs to include cross-talk or di-attenuation effects due to all lidar optics and it is given by:

$$
\delta=\frac{\delta^{*}\left(G_{T}+H_{T}\right)-\left(G_{R}+H_{R}\right)}{\left(G_{R}-H_{R}\right)-\delta^{*}\left(G_{T}-H_{T}\right)}
$$

where $G_{T}, H_{T}, G_{R}, H_{R}$ are four polarization cross-talk correction parameters characterizing both transmitted and reflected polarization channels. Such parameters can be calculated (for details see [4]) and stored in the SCC database as channel options. Finally, when the 


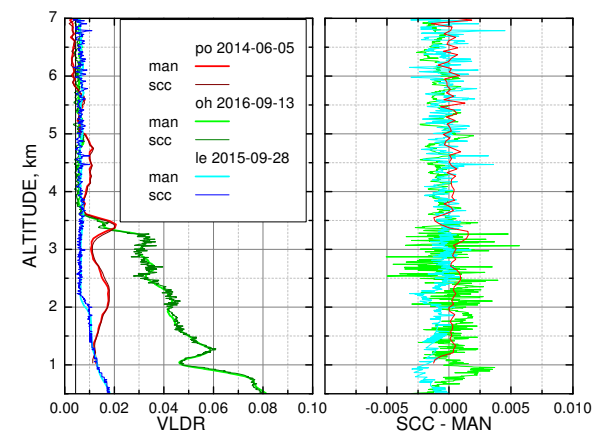

Figure 1: Comparison of VLDR profiles which were derived manually (bold lines) and by SCC (thin lines) for several EARLINET systems.

VLDR is known, it is possible to calculate the PLDR using the following formula:

$$
\delta_{a}=\frac{\left(1+\delta_{m}\right) \delta R-(1+\delta) \delta_{m}}{\left(1+\delta_{m}\right) R-(1+\delta)}
$$

where $\delta_{m}$ is the theoretical value for the molecular linear depolarization ratio and $R$ is the backscatter ratio. In Fig. 1 is shown a comparison between the manually retrieved VLDR profiles and the corresponding ones calculated by the SCC for several lidar systems. The red (blue) profiles were measured in Potenza (Leipzig) by the MUSA (PollyXT) system. The green profiles were measured in Hohenpeißenberg by the EARLINET reference lidar POLIS (light green, bold - manually retrieved) and by RALPH system (dark green, thin - SCC retrieved). Differences between the manually and corresponding SCC retrieved profiles are shown in the right panel.

\subsection{Cloud masking}

The aim of the cloud masking module, still in a testing phase, is to separate cloudy and cloud-free regions from raw, uncalibrated lidar signals. The module treats each submitted measurement file independently, not considering previous analysis, as we cannot be sure that the instrument calibration constant did not change even in a small time period. This approach does not allow us to apply quantitative,

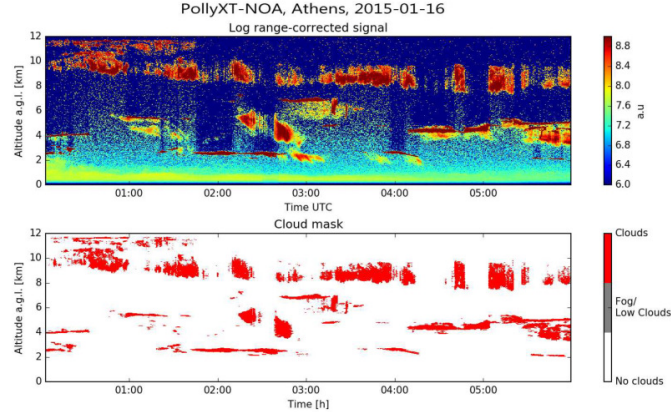

Figure 2: Example output of the cloud masking algorithm on PollyXT lidar measurements.

physically meaningful thresholds, for cloud detection. Therefore the aim of this cloud masking module is to provide an initial estimate of cloud-free regions, and it is not intended for the analysis of cloud properties. Before applying the cloud masking procedure, all signals are pre-processed and normalized using the median value of the logarithm of the range corrected signal in the first few kilometers of the atmosphere. In this way signals from different systems have comparable magnitude, helping the stability of the cloud detection. To separate cloudy and non-cloudy regions we examine two features: a) the presence of sharp edges in the signal, quantified by the Sobel operator, and b) the large time variability of the signal, quantified by the standard deviation of the signal in a moving window. Presently, we assume that we can adequately separate cloudy and cloudfree regions in this feature space using a linear decision boundary. To find the optimal boundary, we constructed a "training" set of measurements where we manually apply a cloud mask, based on visual inspection. Then we use a stochastic linear descent algorithm with logistic loss function to find the optimal linear relationship between the two features that is able to separate the two classes of data.

\subsection{Aerosol Layering}

Aerosol layer detection can be done on highresolution pre-processed data or on the vertical profiles of the aerosol optical properties. Both 
approaches will be implemented in the SCC as separate modules. As first step, the assessment of the performances of the aerosol layer detection based on optical properties is planned. In particular, for a pilot study, a prototype of the detection algorithm has been developed. To allow the application of automatic procedures during both day and night time conditions, only the backscatter coefficient profiles and the related Ångström exponents are considered. The current algorithm first checks at which altitudes a significant aerosol load is present (by defining proper threshold on the value of backscatter coefficients); then, within these ranges, significant gradients of the backscatter coefficient profile are identified and from that layer boundaries are defined. Finally, it is checked if the intensive aerosol properties (Ångström exponent) within the defined layers are nearly constant. If not, the layer is divided into sub layers of nearly constant Ångström exponents. Fig. 3 shows an example of the detection algorithm on a Leipzig measurement case where two distinct aerosol layers were automatically detected. In the near future it is planned to extend the procedure to other intensive properties like lidar ratio and particle depolarization ratio.

\section{CONCLUSIONS}

We have provided an overview of the new SCC products like PLDR, VLDR, cloud masking, and aerosol layering. These new products together with the ones already available (aerosol extinction and backscatter coefficients) allows for a better characterization of atmospheric aerosols. In particular, the quality assured procedures used to calculate the PLDR allows a reliable classification of scatterers in terms of their sphericity. The cloud masking module provides a big improvement in terms of SCC near-real time products availability. The aerosol layering products are useful for a large number of application like micro-physical retrievals or aerosol typing schemes. Finally, the flexibility characterizing the implemented products allows the SCC to be easily extended

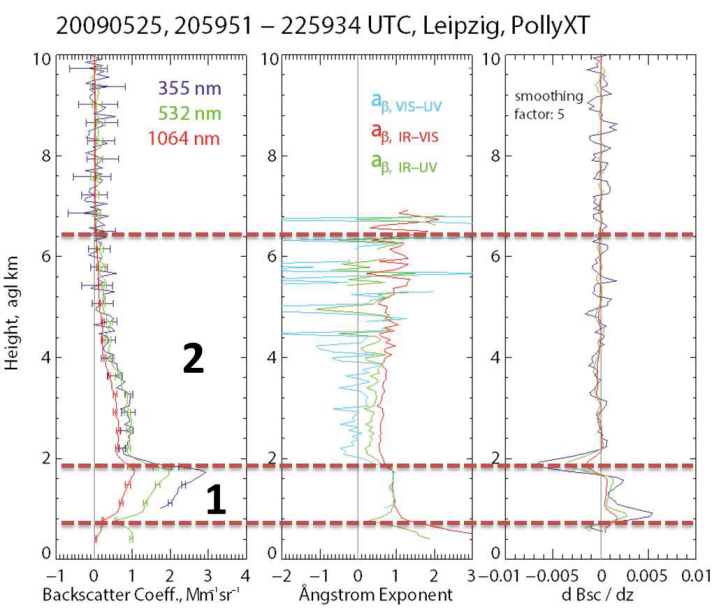

Figure 3: Automatic layer detection algorithm applied on a Leipzig measurement case.

to GALION (GAW Aerosol LIdar Observation Network) to evaluate lidar data of networks different from EARLINET.

\section{ACKNOWLEDGMENTS}

The financial support for EARLINET in the ACTRIS Research Infrastructure Project by the European Union's Horizon 2020 research and innovation programme under grant agreement no 654169 is gratefully acknowledged.

\section{References}

[1] D'Amico, G. et al., 2016: EARLINET Single Calculus Chain - technical - Part 1: Preprocessing of raw lidar data, Atmos. Meas. Tech., 9, (2), 491-507.

[2] Mattis, I. et al., 2016: EARLINET Single Calculus Chain - technical - Part 2: Calculation of optical products, Atmos. Meas. Tech., 9, (7), 3009-3029.

[3] D'Amico, G. et al., 2015: EARLINET Single Calculus Chain - overview on methodology and strategy, Atmos. Meas. Tech., 8, (11), 4891-4916.

[4] Freudenthaler, V. 2016: About the effects of polarising optics on lidar signals and the $\Delta 90$ calibration, Atmos. Meas. Tech., 9, 41814255 . 